\title{
The scaling infrared DSE solution as a critical end-point for the family of decoupling ones
}

\author{
J. Rodríguez-Quintero \\ Dpto. Física Aplicada, Fac. Ciencias Experimentales; Universidad de Huelva, 21071 Huelva; Spain
}

\begin{abstract}
.
Both regular (the zero-momentum ghost dressing function not diverging), also named decoupling, and critical (diverging), also named scaling, Yang-Mills propagators solutions can be obtained by analyzing the low-momentum behaviour of the ghost propagator Dyson-Schwinger equation (DSE) in Landau gauge. The asymptotic expression obtained for the regular or decoupling ghost dressing function up to the order $\mathscr{O}\left(q^{2}\right)$ fits pretty well the low-momentum ghost propagator obtained through the numerical integration of the coupled gluon and ghost DSE in the PT-BFM scheme. Furthermore, when the size of the coupling renormalized at some scale approaches some critical value, the PT-BFM results seems to tend to the the scaling solution as a limiting case.
\end{abstract}

Keywords: Dyson-Schwinger equations, Infrared QCD

PACS: $12.35 . \mathrm{Aw}, 12.38 . \mathrm{Lg}, 12.38 . \mathrm{GC}$

\section{INTRODUCTION}

The low-momentum behaviour of the Yang-Mills propagators derived either from the tower of Dyson-Schwinger equations (DSE) or from Lattice simulations in Landau gauge has been a very interesting and hot topic for the last few years. It seems by now well established that, if we assume in the vanishing momentum limit a ghost dressing function behaving as $F\left(q^{2}\right) \sim\left(q^{2}\right)^{\alpha_{F}}$ and a gluon propagator as $\Delta\left(q^{2}\right) \sim\left(q^{2}\right)^{\alpha_{G}-1}$ (or, by following a notation commonly used, a gluon dressing function as $\left.G\left(q^{2}\right)=q^{2} \Delta\left(q^{2}\right) \sim\left(q^{2}\right)^{\alpha_{G}}\right)$, two classes of solutions may emerge (see, for instance, the discussion of refs. [1, 2]) from the DSE: (i) those, dubbed "decoupling", where $\alpha_{F}=0$ and the suppression of the ghost contribution to the gluon propagator DSE results in a massive gluon propagator (see [3, 4] and references therein); and (ii) those, dubbed "scaling", where $\alpha_{F} \neq 0$ and the lowmomentum behaviour of both gluon and ghost propagators are related by the coupled system of DSE through the condition $2 \alpha_{F}+\alpha_{G}=0$ implying that $F^{2}\left(q^{2}\right) G\left(q^{2}\right)$ goes to a non-vanishing constant when $q^{2} \rightarrow 0$ (see $[5,6]$ and references therein).

Lattice QCD results appear to support only the massive gluon $\left(\alpha_{G}=1\right)$ or scaling solutions (see [7] and references therein), and also pinching technique results (see, for instance, $[8,14]$ and references therein), refined Gribov-Zwanziger formalism (see [9]) or other approaches like the infrared mapping of $\lambda \phi^{4}$ and YangMills theories in ref. [10] or the massive extension of the Fadeev-Popov action in ref. [11] appear to point to. In the present note, we briefly review the work of ref. [13], which extended the previous studies of refs. [1,2, 12], by the analysis of the results obtained by solving the coupled system of Landau gauge ghost and gluon propagators DSE within the framework of the pinching technique in the background field method [14] (PT-BFM)

\section{THE TWO KINDS OF SOLUTIONS OF THE GHOST PROPAGATOR DYSON-SCHWINGER EQUATION}

As was explained in detail in refs. [2, 12, 13], the low-momentum behavior for the Landau gauge ghost dressing function can be inferred from the analysis of the Dyson-Schwinger equation for the ghost propagator (GPDSE). That analysis is performed on a very general ground: one applies the MOM renormalization prescription, $F_{R}\left(\mu^{2}\right)=\mu^{2} \Delta_{R}\left(\mu^{2}\right)=1$, where $\mu^{2}$ is the subtraction point, chooses for the ghost-gluon vertex,

$$
\begin{aligned}
\widetilde{\Gamma}_{v}^{a b c}(-q, k ; q-k) & =i g_{0} f^{a b c}\left(q_{v} H_{1}(q, k)\right. \\
& \left.+(q-k)_{v} H_{2}(q, k)\right)
\end{aligned}
$$

to apply this MOM prescription in Taylor kinematics (i.e. with a vanishing incoming ghost momentum) and assumes the non-renormalizable bare ghost-gluon form factor, $H_{1}(q, k)=H_{1}$, to be constant in the lowmomentum regime for the incoming ghost. Then, the low momentum-behaviour of the ghost dressing function is supposed to be well described by

$$
F_{R}\left(q^{2}\right)=A\left(\mu^{2}\right)\left(\frac{q^{2}}{M^{2}}\right)^{\alpha_{F}}(1+\cdots),
$$


and that of the gluon propagator by

$$
\Delta_{R}\left(q^{2}\right)=\frac{B\left(\mu^{2}\right)}{q^{2}+M^{2}} \simeq \frac{B\left(\mu^{2}\right)}{M^{2}}\left(1-\frac{q^{2}}{M^{2}}+\cdots\right)
$$

and this, after solving asymptotically the GPDSE, finally left us with:

$$
F_{R}\left(q^{2}\right) \simeq\left(\frac{10 \pi^{2}}{N_{C} H_{1} g_{R}\left(\mu^{2}\right) B\left(\mu^{2}\right)}\right)^{1 / 2}\left(\frac{M^{2}}{q^{2}}\right)^{1 / 2}
$$

if $\alpha_{F} \neq 0$; and

$$
\begin{aligned}
F_{R}\left(q^{2}\right) & \simeq F_{R}(0)\left(1+\frac{N_{C} H_{1}}{16 \pi} \bar{\alpha}_{T}(0) \frac{q^{2}}{M^{2}}\left[\ln \frac{q^{2}}{M^{2}}-\frac{11}{6}\right]\right. \\
& \left.+\mathscr{O}\left(\frac{q^{4}}{M^{4}}\right)\right)
\end{aligned}
$$

if $\alpha_{F}=0$, where

$$
\bar{\alpha}_{T}(0)=M^{2} \frac{g_{R}^{2}\left(\mu^{2}\right)}{4 \pi} F_{R}^{2}(0) \Delta_{R}(0) .
$$

It should be understood that the subtraction momentum for all the renormalization quantities is $\mu^{2}$. The case $\alpha_{F} \neq 0$ leads to the so-called scaling solution, where the low-momentum behavior of the massive gluon propagator forces the ghost dressing function to diverge at lowmomentum through the scaling condition: $2 \alpha_{F}+\alpha_{G}=0$ ( $\alpha_{G}=1$ is the power exponent when dealing with a massive gluon propagator). As this scaling condition is verified, the perturbative strong coupling defined in this Taylor scheme [15], $\alpha_{T}=g_{T}^{2} /(4 \pi)$, has to reach a constant at zero-momentum,

$$
\begin{aligned}
\alpha_{T}(0) & =\frac{g^{2}\left(\mu^{2}\right)}{4 \pi} \lim _{q^{2} \rightarrow 0} q^{2} \Delta\left(q^{2}\right) F^{2}\left(q^{2}\right) \\
& =\frac{5 \pi}{2 N_{C} H_{1}}
\end{aligned}
$$

as can be obtained from Eqs. $(3,5)$. The case $\alpha_{F}=0$ corresponds to the so-called decoupling solution, where the zero-momentum ghost dressing function reaches a nonzero finite value and eq. (5) provides us with the first asymptotic corrections to this leading constant. This subleading correction is controlled by the zero-momentum value of the coupling defined in eq. (6), which is an extension of the non-perturbative effective charge definition from the gluon propagator [16] to the Taylor ghostgluon coupling [17].

\section{The "critical" limit}

In ref. [13], the solutions of the coupled DSE system in the PT-BFM scheme (with $H_{1}=1$ for the ghost-gluon vertex), numerically integrated for many values of the coupling at the renormalization point $\mu^{2}$ as a boundary condition, were studied. They were shown to behave asymptotically as eq. (4) predicts for the decoupling DSE solutions. There also appeared to be a critical value of the coupling, $\alpha_{\text {crit }}=\alpha\left(\mu^{2}\right) \simeq 0.182$ with $\mu=10 \mathrm{Gev}$, above which the coupled DSE system does not converge any longer to a solution. As a matter of fact, we know from refs. $[2,13]$ that the scaling solution implies for the coupling

$$
\alpha_{\mathrm{crit}}=\frac{g_{R}^{2}\left(\mu^{2}\right)}{4 \pi} \simeq \frac{5 \pi}{2 N_{C} A^{2}\left(\mu^{2}\right) B\left(\mu^{2}\right)},
$$

where $B\left(\mu^{2}\right)$ and $A\left(\mu^{2}\right)$ defined by Eqs. $(3,4)$. This is also shown in ref. [1], where only the ghost propagator DSE with the kernel for the gluon loop integral is obtained from gluon propagator lattice data. In the analysis of ref. [1], a ghost dressing function solution diverging at vanishing momentum appears to exist and verifies eqs. $(4,8)$, while regular or decoupling solutions exist for any $\alpha<\alpha_{\text {crit }}$. In ref. [13], a more complete analysis is performed: first by studying the solutions for many different values of the coupling, $\alpha=\alpha\left(\mu^{2}\right)$, of a coupled DSE system; and then by showing that the ghost dressing function at vanishing momentum, $F\left(0, \mu^{2}\right)$, is described by the following power behaviour,

$$
F(0) \sim\left(\alpha_{\text {crit }}-\alpha\left(\mu^{2}\right)\right)^{-\kappa\left(\mu^{2}\right)}
$$

where $\kappa\left(\mu^{2}\right)$ is a critical exponent (depending presummably on the renormalization point, $\mu^{2}$ ), supposed to be positive and to govern the transition from decoupling $\left(\alpha<\alpha_{\text {crit }}\right.$ ) to the scaling ( $\alpha=\alpha_{\text {crit }}$ ) solutions; and where we let $\alpha_{\text {crit }}$ be a free parameter to be fitted by requiring the best linear correlation for $\log [F(0)]$ in terms of $\log \left[\alpha_{\text {crit }}-\alpha\right]$. In doing so, the best correlation coefficient is 0.9997 for $\alpha_{\text {crit }}=0.1822$, which is pretty close to the critical value of the coupling above which the coupled DSE system does not converge any more, and $\kappa\left(\mu^{2}\right)=0.0854(6)$. This can be seen in fig. 1 , where the log-log plot of $F_{R}(0)$ in terms of $\alpha_{\text {crit }}-\alpha$ is shown and the linear behaviour with negative slope corresponding to the best correlation coefficient strikingly indicates a zero-momentum ghost propagator diverging as $\alpha \rightarrow \alpha_{\text {crit }}$. Nevertheless, no critical or scaling solution appears for the coupled DSE system in the PTBFM, although the decoupling solutions obtained for any $\alpha<\alpha_{\text {crit }}=0.1822$ seem to approach the behaviour of a scaling one when $\alpha \rightarrow \alpha_{\text {crit }}$. This is well understood in ref. [13], where the gluon propagators obtained from the coupled DSE system in PT-BFM were also found to obey the same critical behaviour pattern as the ghost propagator, when approaching the critical value of the coupling. 


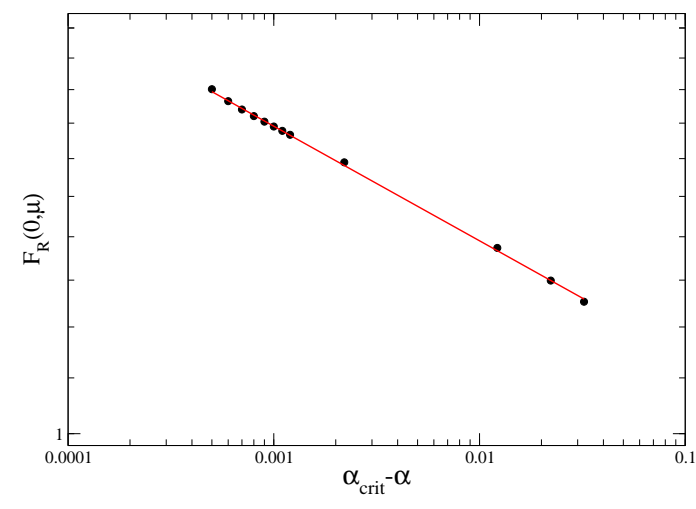

FIGURE 1. Log-log plot of the zero-momentum values of the ghost dressing function, obtained by the numerical integration of the coupled DSE system in the PT-BFM scheme, in terms of $\alpha_{\text {crit }}-\alpha . \alpha=\alpha(\mu=10 \mathrm{GeV})$, the value of the coupling at the renormalization momentum, is an initial condition for the integration; while $\alpha_{\text {crit }}$ is fixed to be 0.1822 , as explained in the text, by requiring the best linear correlation.

\section{CONCLUSIONS}

The ghost propagator DSE, with the only assumption of taking $H_{1}(q, k)$ from the ghost-gluon vertex in eq. (1) to be constant in the infrared domain of $q$, can be exploited to look into the low-momentum behaviour of the ghost propagator. The two classes of solutions named "decoupling" and "scaling" can be indentified and shown to depend on whether the ghost dressing function achieves a finite non-zero constant $\left(\alpha_{F}=0\right)$ at vanishing momentum or not $\left(\alpha_{F} \neq 0\right)$. The solutions appear to be dialed by the size of the coupling at the renormalization momentum which plays the role of a boundary condition for the DSE integration. When applying a model with a massive gluon propagator, the decoupling low-momentum behaviour of the ghost propagator results to be regulated by the gluon mass and the Taylor-scheme effective charge at zero momentum and successfully describes the low-momentum ghost propagator computed trhough the numerical integration of the coupled gluon and ghost DSE in the PT-BFM scheme. The zero-momentum ghost dressing function is then shown to tends to diverge when the value of the coupling dialing the solutions approaches some critical value. Such a divergent behaviour at the critical coupling corresponds to a scaling solution where, if the gluon is massive, $\alpha_{F}=-1 / 2$.

\section{ACKNOWLEDGMENTS}

The author acknowledges the Spanish MICINN for the support by the research project FPA2009-10773 and "Junta de Andalucia" by P07FQM02962.

\section{REFERENCES}

1. Ph. Boucaud, J. P. Leroy, A. L. Yaouanc, J. Micheli, O. Pene and J. Rodriguez-Quintero, JHEP 0806 (2008) 012 arXiv:0801.2721 [hep-ph].

2. Ph. Boucaud, J. P. Leroy, A. Le Yaouanc, J. Micheli, O. Pene and J. Rodriguez-Quintero, JHEP 0806 (2008) 099 [arXiv:0803.2161 [hep-ph]].

3. A. C. Aguilar and J. Papavassiliou, JHEP 0612 (2006) 012; Eur. Phys. J. A 31 (2007) 742; A. C. Aguilar and A. A. Natale, JHEP 0408 (2004) 057.

4. A. C. Aguilar, D. Binosi and J. Papavassiliou, Phys. Rev. D 78 (2008) 025010 [arXiv:0802.1870 [hep-ph]].

5. R. Alkofer and L. von Smekal, Phys. Rept. 353 (2001) 281 [arXiv:hep-ph/0007355]; C. Lerche and L. von Smekal, Phys. Rev. D 65 (2002) 125006 [arXiv:hep-ph/0202194]; D. Zwanziger, Phys. Rev. D 65 (2002) 094039 [arXiv:hepth/0109224]; C. S. Fischer and R. Alkofer, Phys. Lett. B 536 (2002) 177 [arXiv:hep-ph/0202202]; J. M. Pawlowski, D. F. Litim, S. Nedelko and L. von Smekal, Phys. Rev. Lett. 93 (2004) 152002 [arXiv:hep-th/0312324]. M. Q. Huber, R. Alkofer, C. S. Fischer and K. Schwenzer, Phys. Lett. B 659 (2008) 434 [arXiv:0705.3809 [hep-ph]].

6. C. S. Fischer, A. Maas and J. M. Pawlowski, Annals Phys. 324 (2009) 2408 [arXiv:0810.1987 [hep-ph]].

7. A. Cucchieri and T. Mendes, PoS LAT2007 (2007) 297 Phys. Rev. Lett. 100 (2008) 241601; I. L. Bogolubsky, E. M. Ilgenfritz, M. Muller-Preussker and A. Sternbeck, Phys. Lett. B 676 (2009) 69; I. L. Bogolubsky, E. M. Ilgenfritz, M. Muller-Preussker and A. Sternbeck, PoS LAT2007 (2007) 290; A. Sternbeck, E.-M. Ilgenfritz, M. M uller-Preussker and A. Schiller, Nucl. Phys. Proc. Suppl. 140 (2005) 653; AIP Conference Proceedings 756 (2005) 284, [arXiv:hep-lat/0412011]; P. Boucaud et al., [arXiv:hep-ph/0507104 ]; O. Oliveira and P. Bicudo, arXiv:1002.4151 [hep-lat]; V. G. Bornyakov, V. K. Mitrjushkin and M. Muller-Preussker, Phys. Rev. D 81 (2010) 054503

8. J. M. Cornwall, Phys. Rev. D 26, 1453 (1982).

9. D. Dudal, J. A. Gracey, S. P. Sorella, N. Vandersickel and H. Verschelde, Phys. Rev. D 78 (2008) 065047 [arXiv:0806.4348 [hep-th]].

10. M. Frasca, Phys. Lett. B 670 (2008) 73

11. M. Tissier and N. Wschebor, arXiv:1004.1607 [hep-ph].

12. Ph. Boucaud al., Phys. Rev. D 82 (2010) 054007 [arXiv:1004.4135 [hep-ph]]

13. J. Rodriguez-Quintero, [arXiv:1005.4598 [hep-ph]].

14. D. Binosi and J. Papavassiliou, Phys. Rev. D 66 (2002) 111901 [arXiv:hep-ph/0208189]; Phys. Rev. D 77 (2008) 061702 [arXiv:0712.2707 [hep-ph]]; D. Binosi and J. Papavassiliou, Phys. Rev. D 77 (2008) 061702 [arXiv:0712.2707 [hep-ph]].

15. Ph. Boucaud et al., Phys. Rev. D 79 (2009) 014508 [arXiv:0811.2059 [hep-ph]]; A. Sternbeck, K. Maltman, L. von Smekal, A. G. Williams, E. M. Ilgenfritz and M. Muller-Preussker, PoS LAT2007 (2007) 256 [arXiv:0710.2965 [hep-lat]].

16. A. C. Aguilar, D. Binosi and J. Papavassiliou, PoS LC2008 (2008) 050 [arXiv:0810.2333 [hep-ph]].

17. A. C. Aguilar, D. Binosi, J. Papavassiliou and J. Rodriguez-Quintero, Phys. Rev. D 80 (2009) 085018 [arXiv:0906.2633 [hep-ph]]. 